\title{
Soft Magnetorotons and Broken-Symmetry States in Bilayer Quantum Hall Ferromagnets
}

\author{
Stefano Luin ${ }^{\mathrm{a}, \mathrm{b}}$ Vittorio Pellegrini ${ }^{\mathrm{a}}$ Aron Pinczuk ${ }^{\mathrm{b}, \mathrm{c}}$ Brian S. Dennis ${ }^{\mathrm{b}}$ Loren N. Pfeiffer ${ }^{\mathrm{b}}$ \\ Ken W. West ${ }^{b}$ \\ ${ }^{a}$ NEST-INFM and Scuola Normale Superiore, Piazza dei Cavalieri 7, I-56126 Pisa (Italy) \\ ${ }^{\mathrm{b}}$ Bell Laboratories, Lucent Technologies, Murray Hill, New Jersey 07974, USA \\ ${ }^{\mathrm{c}}$ Department of Physics, Department of Applied Physics and Applied Mathematics, Columbia University, New York, New \\ York 10027, USA
}

\begin{abstract}
The recent report on the observation of soft magnetorotons in the dispersion of charge-density excitations across the tunneling gap in coupled bilayers at total Landau level filling factor $\nu_{T}=1$ is reviewed. The inelastic light scattering experiments take advantage of the breakdown of wave-vector conservation that occurs under resonant excitation. The results offer evidence that in the quantum Hall state there is a roton that softens and sharpens markedly when the phase boundary for transitions to highly-correlated compressible states is approached. These findings are interpreted with Hartree-Fock evaluations of the dynamic structure factor. The model includes the effect of disorder in the breakdown of wave-vector conservation and resonance enhancement profiles within a phenomenological approach. These results link the softening of magnetorotons to enhanced excitonic Coulomb interactions in the ferromagnetic bilayers.
\end{abstract}

Key words: Quantum Hall effect, light scattering, coupled bilayers PACS: 73.43.Lp, 78.30.-j, 73.21.-b

\section{Introduction}

Two-dimensional electron systems in quantizing magnetic fields exhibit a variety of collective phases [1]. The additional degree of freedom associated to layer occupation makes bilayer systems at total Landau level filling factor $\nu_{T}=1$ particularly interesting [2]. At this filling factor a phase transition exists between incompressible and compressible phases (see Fig.1(c)) [3]. The incompressible phases are stable at large tunnelling gaps $\Delta_{\text {SAS }}$ and/or low interlayer distances $\mathrm{d}$. Compressible phases, characterized by the absence of the quantum Hall signature, emerge at large d and low $\Delta_{\text {SAS. }}$.

The phase transition is finely tuned by the interplay between $\Delta_{\text {SAS }}$ with intra- and inter-layer Coulomb interactions. In the incompressible phases the different dependences of depolarization and excitonic terms on wave-vector produce, at $|\mathbf{q}|=q>0$, a magnetoroton (MR) minimum in the dispersion of the chargedensity tunnelling excitation (CDE). In current theories the incompressible-compressible phase transition is linked to an instability due to softening of a magnetoroton of CDE modes[4,5,6]. Recent experimental results on ground state properties close to the phase boundary were focused on Coulomb drag and inter- 
layer tunnelling at very low values of $\Delta_{\mathrm{SAS}}[7,8,9,10]$. These studies suggested the appearance of a Goldstone mode in the incompressible phase and offered evidence of superfluid behavior.

Experiments that probe dispersive collective excitations and their softening as a function of $\Delta_{\text {SAS }}$ and $d$ could provide direct evidence of the impact of excitonic terms of interactions in the quantum phase transitions of the bilayers at $\nu_{T}=1$. In previous experiments, resonant inelastic light scattering methods were employed to access $q \approx 0$ soft tunneling spin excitations at even values of $\nu_{T}[11,12,13]$.

In the work reported here resonant inelastic light scattering is employed to probe the low-energy charge excitations of bilayer quantum Hall (QH) ferromagnets at $\nu_{T}=1$ in states close to a compressibleincompressible phase boundary [14]. These experiments seek direct evidence of soft MR modes with $q \sim l_{B}^{-1}\left(l_{B}\right.$ is the magnetic length), and attempt to uncover the impact of excitonic terms of interactions in the quantum phase transitions of the $\nu_{T}=1$ bilayer systems.

These resonant inelastic light scattering experiments take advantage of breakdown of wave-vector conservation that occurs in the $\mathrm{QH}$ state due to residual disorder. This aspect of light scattering methods offers experimental access to critical points in the mode dispersion such as the one at the MR minimum. To gain fur-

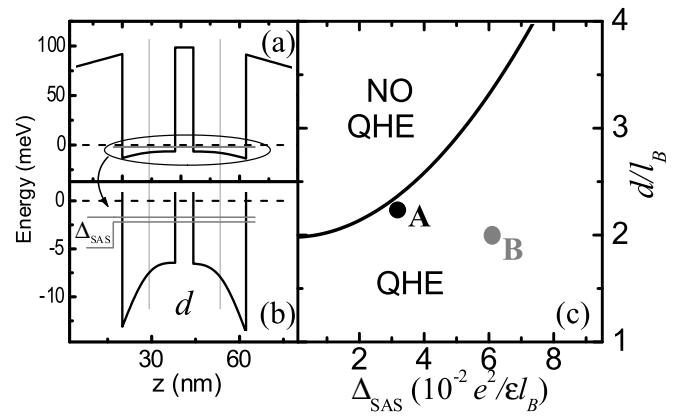

Figure 1. (a): Self-consistent calculations of conduction band diagram of the double quantum well. $d$ is the center-to-center distance between the two wells. The lowest symmetric and antisymmetric levels are shown as a dotted horizontal line. (b) Enlargement of the self-consistent calculation of the conduction band diagram of the double quantum well close to the tunneling gap. The dashed horizontal line marks the chemical potential. (c) Phase diagram of coupled bilayers at $\nu_{T}=1 . l_{B}$ is the magnetic length and $\varepsilon$ the dielectric constant. ther insights from the data, the results are compared to time-dependent Hartree-Fock (TDHF) calculations [15] of light scattering intensities. The evaluations incorporate effects of breakdown of wave-vector conservation [16], as well as the resonant enhancement. This simplified model successfully reproduces both MR energies and light scattering lineshapes. This success indicates that the sharpening of the MR spectral lineshape follows from remarkable changes in the mode dispersion and matrix elements near the incompressiblecompressible phase boundary. These results uncover significant evidence that softening of rotons play major roles in the phase transitions of bilayers at $\nu_{T}=1$, and suggest a leading role for excitonic Coulomb interactions in transitions between these highly correlated phases.

\section{Experimental results}

We present results obtained in two modulationdoped double quantum wells (DQWs) grown by molecular beam epitaxy. They consist of two $18 \mathrm{~nm}$ GaAs wells separated by an undoped $\mathrm{Al}_{0.1} \mathrm{Ga}_{0.9} \mathrm{As}$ barrier (7.5 nm for sample A and $6.23 \mathrm{~nm}$ for sample B). Figure 1(a) shows the self-consistent profile of the bottom of the conduction band in the DQWs. The dotted line represent the energy of the lowest symmetric and antisymmetric states. Their splitting, $\Delta_{\text {SAS }}$ is below $1 \mathrm{meV}$ as shown in Fig. 1(b). $d$ is the center-to-center distance between the two wells.

The samples were designed to have the relatively high $\Delta_{\text {SAS }}$ of $0.32 \mathrm{meV}$ in sample $\mathrm{A}$ and $0.58 \mathrm{meV}$ in sample B. Magneto-transport confirms that both samples are in the quantum Hall side of the $\nu_{T}=$ 1 incompressible-compressible phase diagram as shown in Fig. 1(c) (filled circles). Detailed analysis of the magneto-transport features, however, is hindered by the large parallel conduction found at high magnetic fields. Measured total sheet densities are $1.2 \times 10^{11} \mathrm{~cm}^{-2}$ in sample A and $1.1 \times 10^{11} \mathrm{~cm}^{-2}$ in sample $B$ with mobilities larger than $10^{6} \mathrm{~cm}^{2} / \mathrm{Vs}$. Inelastic light scattering spectra are obtained in a back-scattering geometry with light propagating along the magnetic field, almost perpendicular to the 2DEG plane. Samples are mounted in a ${ }^{3} \mathrm{He} /{ }^{4} \mathrm{He}$ dilution cryo-magnetic system with optical windows, at a 
small tilt angle (20 degrees) with respect to the incoming laser light. Accessible temperatures are in the range $50 \mathrm{mK}-1.4 \mathrm{~K}$. For these measurements the optical emission of a dye laser is tuned to a frequency $\omega_{I}$ close to the fundamental interband transitions of the DQW. Incident power densities are kept below $10^{-4} \mathrm{~W} / \mathrm{cm}^{2}$, and spectra are recorded using a double monochromator, CCD multichannel detection and spectral resolution of $15 \mu \mathrm{eV}$.

Figure 2(a) shows resonant inelastic light spectra of CDE modes in sample A with conventional subtraction of the background due to the laser and to the main magneto-luminescence. The results are obtained at three different laser wavelengths (indicated in the upper part of Fig. 2) and display clearly the three bands of $\mathrm{CDE}$ collective modes. In addition to the CDE at $q \approx 0(\mathrm{C} 0)$ at $\sim 1.08 \mathrm{meV}$, two lower-energy excitations are clearly seen. The lowest energy mode at $\sim$

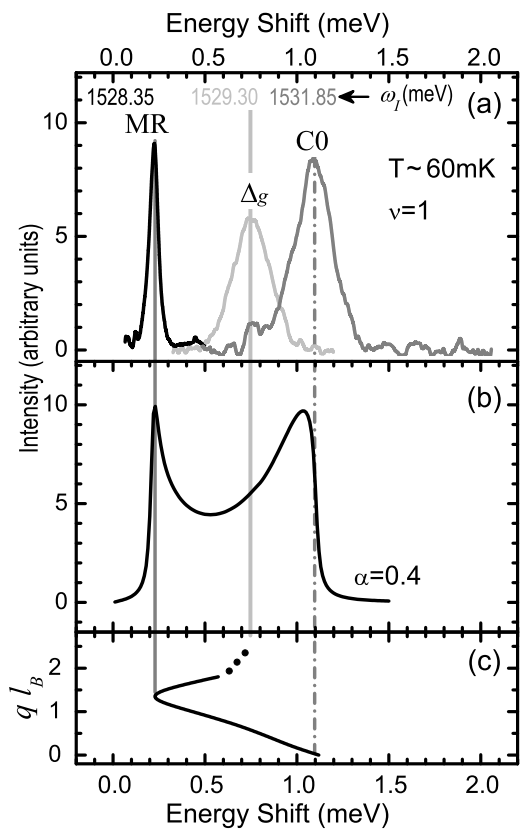

Figure 2. (a) Resonant Inelastic light scattering at $\mathrm{T}=60 \mathrm{mK}$. The magnetoroton $(\mathrm{MR})$ and $\mathbf{q} \approx 0(\mathrm{C} 0)$ and $\mathbf{q} \rightarrow \infty\left(\Delta_{g}\right)$ charge-density wave peaks are shown at three different incident photon energies (reported in the figure). Background due to magneto-luminescence and laser is subtracted. (b) Time-dependent Hartree-Fock (TDHF) calculation of the dynamic strcuture factor as a function of energy for a chosen value of $\alpha=0.4$ (see text for more details). (c) Collective mode dispersion within TDHF. Dotted line is the extrapolation at high values of in-plane wave-vectors.
$0.22 \mathrm{meV}$ is assigned to the MR critical point in the dispersion. The MR in Fig. 2(a) is extremely narrow, with a full width at half maximum (FWHM) of $\sim 0.06 \mathrm{meV}$, which is a factor of three smaller than the FWHM of the $\mathrm{C} 0$ band. The peak at $\sim 0.75 \mathrm{meV}$, labelled $\Delta_{g}$, is the large wavevector CDE excitation. Interpretation of these peaks as the $\Delta_{g}$ and MR modes is confirmed by their marked sensitivity on deviations of magnetic field values from $\nu_{T}=1$ in a manner that is similar to the QH states.

In the following we further corroborate the assignement of MR peak and show how the breakdown of wavevector conservation can lead to manifestations of collective CDE modes at finite $\mathbf{q}$ in the inelastic light scattering spectra. To this end we first recall that intensities of inelastic light scattering is proportional, in first approximation, to the dynamic structure factor $S(q, \omega ; \alpha)$. Here $\alpha$ is a phenomenological broadening parameter in wave-vector space, used to include the effect of disorder in the breakdown of wavevector conservation. This model was first introduced by Marmorkos and Das Sarma [16]. Within this model we have:

$$
S(q, \omega ; \alpha) \sim \frac{\alpha}{\pi l_{B}} \int d q^{\prime} \frac{S\left(q^{\prime}, \omega\right)}{\left(q-q^{\prime}\right)^{2}+\left(\alpha l_{B}^{-1}\right)^{2}},
$$

where $S\left(q^{\prime}, \omega\right)$ is the electronic dynamic structure factor in the translation invariant system. $S\left(q^{\prime}, \omega\right)$ for charge-density excitations across the symmetricantisymmetric gap is given by:

$$
S(q, \omega) \propto \frac{|M(q)|^{2} \omega_{C}(q) \omega \Gamma}{\left[\omega^{2}-\omega_{C}^{2}(q)\right]^{2}+\omega^{2} \Gamma^{2}},
$$

where $\Gamma$ is a homogeneous broadening, $\omega_{C}(q)$ the dispersion of tunneling $\mathrm{CDE}$ and $|M(q)|^{2}$ is a matrix element that acts as an oscillator strength for inelastic light scattering by collective excitations. Figure 2(b) shows the calculated spectra at $\alpha=0.4$. This value yields an effective length scale of $3 \cdot l_{B}$ for the disorder potential involved in the breakdown of wave-vector conservation at $\nu_{T}=1$. The calculated CDE mode dispersion is shown in Fig. 2(c). As expected the calculated dispersion displays a deep magneto-roton. The predicted energies of the CDE at $q=0(\mathrm{C} 0)$ and the MR are consistent with the experimental results. The evolution of this soft MR with changes in density, $\Delta_{\mathrm{SAS}}$, and $d$ can unravel the nature of the phase transition at $\nu_{T}=1$ and will be the subject of future works. We note that the TDHFA being a mean-field theory 


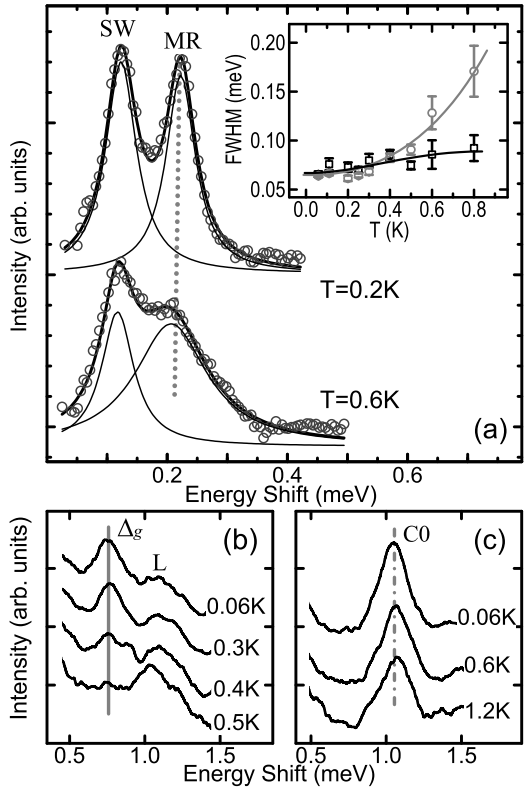

Figure 3. (a) Open circles show the temperature dependence of inelastic light scattering spectra in sample A after background subtraction. The solid lines are results of fits with two lorentzians. Inset: temperature dependence of the full width at half maximum (FWHM) for the spin-wave (SW) peaks at the Zeeman (black empty squares) and the magnetoroton (MR) peaks (gray empty circles). The solid lines are guides for the eyes and the error bars are standard deviations for results on different measurements and with different background subtractions. (b) Temperature dependence of the $q \rightarrow \infty$ excitation $\Delta_{g} ; L$ labels a magneto-luminescence peak. (c) Temperature dependence of the $q=0$ tunneling excitation $C 0$.

fails to reproduce the collective mode dispersion when $q \gg l_{B}^{-1}$. That part of the dispersion (dotted line in Fig. 2(c)) was not included in the calculations.

The temperature dependences of the three excitations shown in Fig. 2 are reported in Fig. 3. Panel (a) of Fig. 3 shows two representative spectra in which both spin-wave (SW) at the Zeeman gap and MR mode are observed. The linewidth of the two excitations have very different temperature dependence as shown in the inset to Fig. 3(a). FWHM values are obtained by Lorentzian lineshape fits [solid lines in Fig. 3(a)].

We note that for temperatures above $0.8 \mathrm{~K}$ the MR mode can no longer be observed while minor changes occur in the SW peak. Similar temperature dependences characterize the $q \rightarrow \infty \Delta_{g}$ mode (see panel b of Fig. 3) and the $\nu_{T}=1 \mathrm{QH}$ effect in magneto-

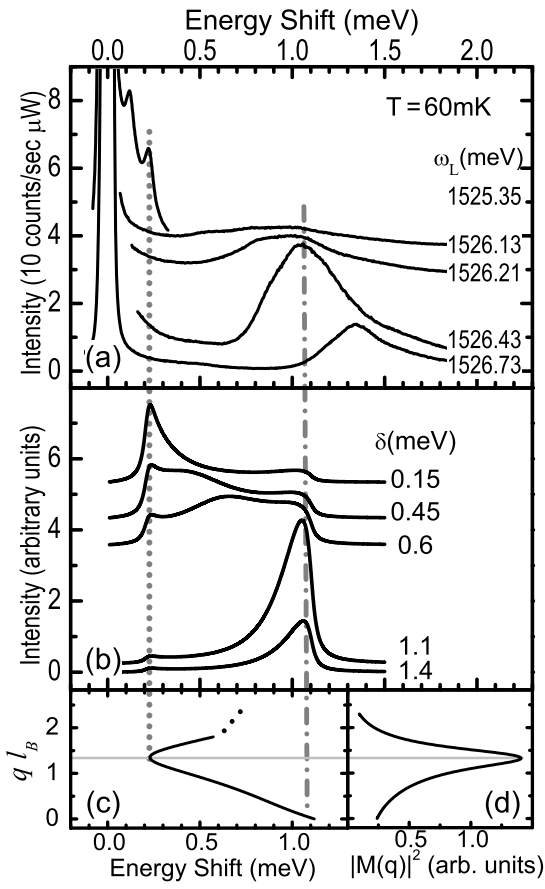

Figure 4. (a) Resonant inelastic light scattering spectra of charge-density excitations (CDEs) in sample A at filling factor $\nu_{T}=1$. The bands were measured with the incident photon energy $\omega_{L}$ indicated in meV. (b) Calculated dynamic structure factor including both breakdown of wave-vector conservation and resonant enhancement (see text and Eqs. 1,3). (c) Calculated dispersion of CDE modes. (d) Calculated inelastic light scattering "matrix element". Vertical lines show the peak position of CDE modes C0 and MR. The horizontal line in (c) and (d) marks the MR wave vector.

transport (not shown). The characteristic temperature for this behavior is here almost one-order of magnitude smaller than $\Delta_{g}$ and more than a factor of three below the MR energy in sample A. On the other end, the $q \sim 0$ ( $\mathrm{C} 0)$ mode is temperature-independent up to $1.4 \mathrm{~K}$ (see panel c of Fig. 3 ).

Figure 4 reports a detailed comparison between experimental inelastic light scattering spectra of sample A as a function of laser frequency (a) and calculated spectra that include the effect of the resonant enhancement profile (b). The latter can be modelled by replacing the dynamic structure factor in (2) with: 


$$
\begin{aligned}
& S(q, \omega ; \delta) \propto \\
& \left\{\left[\omega^{2}-\omega_{C}^{2}(q)\right]^{2}+\omega^{2} \Gamma^{2}\right\}\left[\gamma^{2}+\left(\delta-\omega_{C}(q)\right)^{2}\right]
\end{aligned}
$$

where $\delta$ is the position and $\gamma$ is the width of the resonance. These two parameters can be related to the energy shift from the laser and the width of the luminescence caused by the same interband transition responsible for the outgoing resonant enhancement in the light scattering process. In order to facilitate the comparison between experiment and theory we plot in panel (c) the energy dispersion of the CDE mode shown in Fig.2. Panel (d) of Fig. 4 displays the matrix element $|M(q)|^{2}$ that enters the dynamic structure factor. Large value of $|M(q)|^{2}$ at MR wavevector, compared to that of sample B and shown in Fig. 5, and its sharpness explain the significant manifestations of MRs in the light scattering spectra of sample A.

In order to emphasize the connection between the soft and sharp MR modes observed in sample A and the instability of incompressible quantum Hall state, we studied a second sample far from the phase boundary (sample B in Fig. 1(c)). Figure 5 (a) shows the light scattering spectra observed in sample $\mathrm{B}$. The $\mathrm{C} 0$ band has similar energy and width of that of sample A. It has, in addition, a marginal field dependence and also occur in spectra obtained at $\mathrm{B}=0$. The manifestation of the MR structure, on the other end, is remarkably different from what observed in sample A: it appears as a weak shoulder with a cutoff at $0.65 \mathrm{meV}$. The resonant enhancement profile measured in sample B and shown in Fig. 5(a) reveals a characteristic outgoing resonance with the higher optical interband transition of the luminescence peak labelled $\mathrm{L}$.

The TDHF calculations of the dynamic structure factor (Eqs. 1 and 3) and of the mode dispersion and oscillator strength are reported in Fig. 5 panels b,c,d, respectively. The relatively broad and less intense structure of $|M(q)|^{2}$ explains the weaker manifestation of MRs in the spectra of sample B. These results thus offer the framework to understand energy position, width and relative intensities of the collective modes observed in the resonant inelastic light scattering experiments in the two samples.

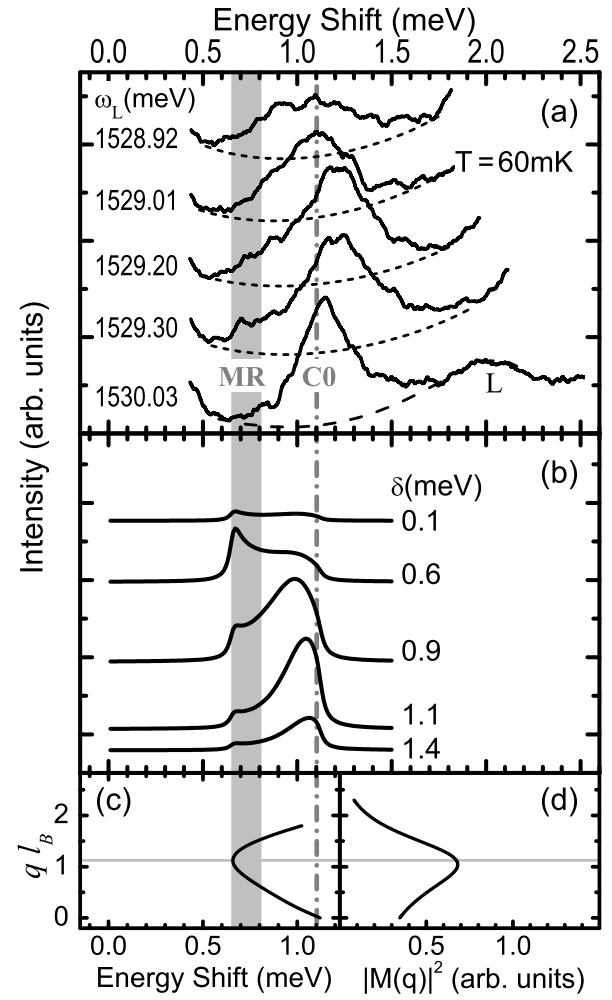

Figure 5. Same as in Fig. 4 but for sample B. (a) Resonant inelastic light scattering spectra of CDEs in sample B at $\nu_{T}=1$. The bands were measured with the incident photon energy $\omega_{L}$ indicated in meV. (b) Calculated dynamic structure factor including both breakdown of wavevector conservation and resonant enhancement (see text and Eq. 2). (c) Calculated dispersion of CDE modes. (d) Calculated inelastic light scattering "matrix element". Vertical lines show the peak position of CDE modes C0 and MR. The horizontal line in (c) and (d) is at the MR wave vector.

\section{Discussion and Conclusion}

Our experiments succeeded in detecting the lowlying magnetoroton minimum in the electron bilayers in AlGaAs/GaAs double quantum wells at total filling factor $\nu_{T}=1$. These results are intriguing and show that the MR peak softens and sharpens markedly when the phase boundary for transitions to highly correlated compressible states is approached. The observed different temperature-dependence behavior of SW and MR excitations shown in Fig. 3 is also intriguing. In previous works $[17,18]$ an anomalous behavior in thermallyactivated magneto-transport was interpreted as evi- 
dence for a finite-temperature transition towards an uncorrelated state. The evolution of the MR linewidth shown in the inset of Fig. 3(a) supports this conclusion. From the smooth increase of the MR linewidth with temperature we may infer that thermal fluctuations could destroy the incompressible state and trigger a transition at finite temperature. It is worth noting that a similar temperature behavior characterizes roton excitations in superfluid ${ }^{4} \mathrm{He}[19,20]$.

In this paper we also provided a detailed description of resonant enhancement profiles and collectivemode lineshapes in the resonant inelastic light scattering spectra based on a phenomenological model that incorporates the disorder-induced breakdown of wavevector conservation within a time-dependent HartreeFock approach.

The results of the experiments discussed in this paper confirm that soft rotons play major roles in the incompressible-compressible phase transition and suggest a leading role of excitonic Coulomb interactions in these transitions $[4,6,14,15]$. The results support the picture that the ground state of the bilayers at $\nu_{T}=1$ evolves towards a broken-symmetry state caused by the excitonic collapse of the energy of tunneling excitations at finite wave-vector[4,6]. The marked narrowing of the MR band and its interpretation within a timedependent Hartree-Fock approximation suggest that the new ground state might be characterized by a roton wave-vector $q_{R} \sim l_{B}{ }^{-1}$.

The results presented here create venues for further light-scattering experiments at $\nu_{T}=1$ that seek definite links between soft modes with the incompressiblecompressible phase boundary.

\section{Acknowledgements}

We are grateful to S. Das Sarma, E. Demler, S.M. Girvin, A.h. MacDonald, S.H. Simon and D.W. Wang for significant discussions. This work was supported by the Nanoscale Science and Engineering Initiative of the National Science Foundation under NSF Award Number CHE-0117752, by a research grant of the W. M. Keck Foundation, by CNR (Consiglio Nazionale delle Ricerche), by INFM/E (Istituto Nazionale per la Fisica della Materia, section E), and by the Italian Ministry of University and Research (MIUR)
References

[1] R. E. Prange, S. M. Girvin (Eds.), The Quantum Hall Effect, Springer-Verlag, New York, 1987.

[2] S. M. Girvin, A. H. MacDonald, Ch. 5, in: Das Sarma and Pinczuk [21], p. 161.

[3] J. P. Eisenstein, Ch. 2, in: Das Sarma and Pinczuk [21], p. 37.

[4] L. Brey, Phys. Rev. Lett. 65 (1990) 903.

[5] L. Brey, Phys. Rev. B 47 (1993) 4585.

[6] A. H. MacDonald, P. M. Platzman, G. S. Boebinger, Phys. Rev. Lett. 65 (1990) 775.

[7] M. Kellogg, I. B. Spielman, J. P. Eisenstein, L. N. Pfeiffer, K. W. West, Phys. Rev. Lett. 88 (2002) 126804.

[8] M. Kellogg, J. P. Eisenstein, L. N. Pfeiffer, K. W. West (2002).

[9] I. B. Spielman, J. P. Eisenstein, L. N. Pfeiffer, K. W. West, Phys. Rev. Lett. 84 (2000) 5808.

[10] I. B. Spielman, J. P. Eisenstein, L. N. Pfeiffer, K. W. West, Phys. Rev. Lett. 87 (2001) 036803.

[11] V. Pellegrini, A. Pinczuk, B. S. Dennis, A. S. Plaut, L. N. Pfeiffer, K. W. West, Phys. Rev. Lett. 78 (1997) 310.

[12] V. Pellegrini, A. Pinczuk, B. S. Dennis, A. S. Plaut, L. N. Pfeiffer, K. W. West, Science 281 (1998) 799.

[13] V. Pellegrini, A. Pinczuk, Solid State Comm. 119 (2001) 301.

[14] S. Luin, V. Pellegrini, A. Pinczuk, B. S. Dennis, L. N. Pfeiffer, K. W. West, Phys. Rev. Lett. 90 (2003) 236802.

[15] Daw-Wei Wang, S. Das Sarma, E. Demler, B. I. Halperin, Phys. Rev. B 66 (2002) 195334.

[16] I. K. Marmorkos, S. Das Sarma, Phys. Rev. B 45 (1992) 13396.

[17] T. S. Lay, Y. W. Suen, H. C. Manoharan, X. Ying, M. B. Santos, M. Shayegan, Phys. Rev. B 50 (1994) 17725(R).

[18] M. Abolfath, R. Golestanian, T. Jungwirht, Phys. Rev. B 61 (2000) 4762 .

[19] H. R. Glyde, A. Griffin, Phys. Rev. Lett. 65 (1990) 1454.

[20] E. C. Svensson, W. Montfrooij, I. M. de Schepper, Phys. Rev. Lett. 77 (1996) 4398.

[21] S. Das Sarma, A. Pinczuk (Eds.), Perspectives in Quantum Hall Effect, Wiley, New York, 1997. 